\section{Kidney \\ Blood Pressure Research}

\title{
Long-Term Renal and Cardiac Outcomes after Stenting in Patients with Resistant Hypertension and Atherosclerotic Renal Artery Stenosis
}

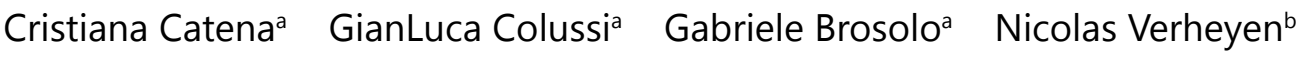 \\ Marileda Novello ${ }^{a}$ Nicole Bertin ${ }^{a} \quad$ Alessandro Cavarape ${ }^{a}$ Leonardo A. Sechi ${ }^{a}$ \\ aHypertension Unit, Internal Medicine, Department of Medicine, University of Udine, Udine, Italy; \\ bDepartment of Cardiology, Medical University of Graz, Graz, Austria
}

\section{Key Words}

Hypertensive nephroangiosclerosis $\bullet$ Renal ultrasound $\bullet$ Fibrinogen $\bullet$ D-dimer $\bullet$ Prothrombotic state.

\begin{abstract}
Background/Aims: Atherosclerotic renal artery stenosis (ARAS) is frequently detected in patients with resistant hypertension (RHTN), but the evidence supporting the utility of renal revascularization in these patients is limited. This prospective, observational study investigates the outcomes of renal stenting in patients with RHTN and hemodynamically significant ARAS. Methods: Fifty-four patients with RHTN were selected because of angiographic evidence of ARAS $>70 \%$ and were followed for 4 years after renal stenting. Renal function and echocardiographic variables were assessed at baseline and during follow-up. Results: Blood pressure decreased rapidly after renal stenting and was normalized in $67 \%$ of patients at six months, with significant reduction in the number of antihypertensive drugs. Creatinine clearance increased in $39 \%$ of patients, decreased in $52 \%$, and remained stable in the remaining $9 \%$, with an average value that had a nonsignificant decrease during follow-up. Urinary albumin excretion did not change throughout the study. After 4 years, left ventricular (LV) wall thickness and concentric geometry decreased significantly and variables of LV diastolic function improved. Conclusion: In patients with RHTN, stenting of hemodynamically significant ARAS decreases blood pressure, preserves renal function in a substantial proportion of patients, and improves LV structure and function, suggesting the opportunity for timely identification of ARAS in these patients.




\section{Kidney Blood Pressure Research}

Catena et al.: Effects of Renal Revascularization

\section{Introduction}

Hypertension is defined as resistant to treatment when two antihypertensive agents plus a diuretic at adequate doses fail to lower blood pressure values below 140/90 mm Hg. Resistant hypertension has a prevalence that approximates $10 \%$ within the overall hypertensive population [1] and is associated with a relatively more severe cardiac and renal damage [2,3]. Resistant hypertension might originate from different conditions including characteristics of lifestyle, obstructive sleep apnoea, advanced hypertensive organ damage, and previously undetected forms of secondary hypertension [4]. Among the latter conditions, chronic renal failure, primary aldosteronism and renovascular hypertension (RVH) caused by atherosclerotic renal artery stenosis (ARAS) are the most frequent. The prevalence of ARAS in the elderly population is comprised from 5 to $10 \%$ [5] and steadily increases in patients undergoing different types of angiographic examinations [6]. In addition to RVH, a possible consequence of ARAS is ischemic nephropathy that might be a relevant cause of renal failure [7] and need for dialysis [8]. For these reasons, timely identification and correction of ARAS might modify the natural history of the disease with possible benefits for both renal and cardiovascular outcomes [9]. However, while a multiplicity of observational studies on revascularization of stenotic renal arteries reported a significant reduction in blood pressure and plasma creatinine, randomized controlled trials that compared renal artery angioplasty with stenting to conservative treatment did not meet the expectations as they could not demonstrate any benefit on renal and cardiovascular outcomes [10]. These randomized trials have been criticized on several grounds and principally for inadequate selection of patients [11]. Indeed, the majority of these studies enrolled patients with a relatively mild degree of renal arterial narrowing or excluded patients at higher cardiovascular risk such as those with resistant hypertension. Although ARAS is frequently detected in patients with resistant hypertension [12], the evidence supporting the clinical utility of renal artery revascularization in these patients is limited because none of the previous studies focused on these specific patients and data currently available originate mostly from subgroup analyses. Moreover, only a few studies have examined in these patients the possible benefits of renal artery revascularization on hypertension-related cardiac changes and albuminuria. The aim of the present study was therefore to investigate prospectively and in the long-term the clinical, cardiac, and renal outcome of renal revascularization in patients with resistant hypertension and angiographic evidence of hemodynamically significant ARAS.

\section{Materials and Methods}

\section{Patients}

Fifty-four patients (age 69 \pm 9 y.; 39 males, 15 females) with resistant hypertension and hemodynamically relevant ARAS detected at renal artery angiography were included in a long-term, observational, prospective study on the effects of renal revascularization with angioplasty and stenting. Patients were consecutively recruited between January 2002 and December 2011 among patients with hypertension resistant to treatment who were referred for evaluation at the Hypertension clinic of the University of Udine. Blood pressure was measured in the sitting position by a validated automated device (Omron M6, OMRON Healthcare Co., Kyoto, Japan) after each patient had been supine for $15 \mathrm{~min}$ and the average of three readings obtained in 5 min was recorded [13]. Resistant hypertension was defined according to established guidelines when systolic blood pressure was $\geq 140 \mathrm{~mm} \mathrm{Hg}$ and/or diastolic blood pressure was $\geq 90 \mathrm{~mm} \mathrm{Hg}$ in patients who were treated with three or more different antihypertensive agents that included a diuretic, at appropriate doses [13]. Predefined exclusion criteria were age younger than 40 years or older than 80 years, renal failure with 24 -hour creatinine clearance $\leq 30 \mathrm{ml} / \mathrm{min} / 1.73 \mathrm{~m}^{2}$, urinary protein excretion $\geq 0.5$ $\mathrm{g} /$ day, congestive heart failure, and any other known renal disease. In all patients, pseudo-resistance (nonadherence to medication, white-coat hypertension, lifestyle) was ruled out with standardized procedures. To investigate the cause of resistant hypertension, all patients seen at the clinic are routinely screened with 


\section{Kidney Blood Pressure Research}

Catena et al.: Effects of Renal Revascularization

clinical and laboratory testing that include analysis of medical records and adherence to treatment, physical examination, urine analysis, blood biochemistries, duplicate measurements of 24-hour creatinine clearance and urinary sodium excretion, plasma active renin and aldosterone, urinary cortisol and catecholamines, electrocardiogram, echocardiography, polysomnography, renal ultrasound with duplex Doppler examination of intrarenal vessels, and renal angio-CT scan or angio-MRI [14]. Renal angiography was performed in all patients in whom increased intrarenal duplex systolic velocity was detected and/or renal artery stenosis was demonstrated either at angio-MRI or angio-CT scan [15]. Patients were classified as smokers if they had smoked for at least 5 years, and up to 1 year before the study. Dyslipidemia was defined by a non-HDL cholesterol $\geq 130 \mathrm{mg} / \mathrm{dl}$ and/or as current use of lipid-lowering medication [16]. Prevalence of coronary heart, cerebrovascular, and peripheral artery disease was also defined in all patients, as previously reported [17].

\section{Prospective study}

Hemodynamically significant ARAS was defined by angiographic demonstration of renal artery stenosis $>70 \%$ in one or more major renal vessels [18] and was detected in 54 patients. Patients with lesser degrees of renal artery narrowing were excluded. No patient had angiographic characteristics of fibromuscular dysplasia or other types of renal artery disease. All the patients included in the prospective study were white, lived in the north-east of Italy, and were representative of the hypertensive population in this area [19]. Patients underwent renal artery revascularization by performing angioplasty with bare metal stenting of the stenotic renal artery. All procedures were performed by femoral approach according to standard interventional techniques. In patients with bilateral stenoses, both vessels were treated in a single procedure. Procedural success was defined as residual stenosis of less than $20 \%$. Before the procedure, combined oral antiplatelet therapy was begun with aspirin (325 mg) and clopidogrel (300 mg). All patients were subsequently assigned to continuous treatment with aspirin (125 mg q.d.) with addition of clopidogrel (75 mg q.d.) for the first 6 months. Clinical and biochemical evaluations were performed after revascularization at 3 weeks, 6 months and 4 years. Antihypertensive treatment was adjusted to reach blood pressure values $\leq 140 / 90 \mathrm{~mm} \mathrm{Hg}$ in quarterly visits during the first year and in yearly visits thereafter. Angiotensin-converting enzyme inhibitors or angiotensin II-receptor antagonists were used as the initial therapeutic choice with subsequently ordered addition of a thiazide diuretic, a dihydropyridine calcium-channel blocker, and a beta-blocker. The study was conducted in accordance with the principles of the Declaration of Helsinki and received approval from the Institutional Review Board. Informed consent was obtained from all patients.

\section{Echocardiography}

Cardiac ultrasound examination was performed by the same experienced operator who was unaware of the patients' clinical and laboratory characteristics [20]. Measurements of left ventricular (LV) internal dimensions and wall thickness were obtained with the patient in the partial left decubitus position with a commercial machine (Aplio CV, Toshiba Medical System, Tokyo, Japan) and a $2.5 \mathrm{MHz}$ transducer, under bi-dimensional cross-sectional control and simultaneous electrocardiographic tracing. The LV geometric pattern was defined by the ratio of end-diastolic posterior LV wall thickness to one-half end-diastolic LV internal dimension (RWT). LV mass index (LVMI) was calculated by the Penn Convention formula and normalized for body surface area. Systolic function was estimated by the ejection fraction (EF) and both endocardial and midwall fractional shortening (FS), with the latter taking into account epicardial migration of the midwall during systole. Pulsed Doppler recordings at the level of the mitral valve tips were obtained from apical four-chambers scans to measure early and late wave diastolic filling velocities, their ratio (E/A ratio), early-wave deceleration time, and isovolumic relaxation time.

\section{Laboratory measurements}

A sample of venous blood was obtained from each patient in the sitting position in the morning after an overnight fast and without venous stasis. Blood was collected into silicone-treated glass tubes, where it was mixed with $10 \%$ of its volume of $0.1 \mathrm{mmol} / \mathrm{l}$ tri-sodium citrate and immediately centrifuged at $1,700 \mathrm{~g}$ for $20 \mathrm{~min}$ at $4^{\circ} \mathrm{C}$. Plasma was separated and frozen at $-80^{\circ} \mathrm{C}$ until being assayed, usually within one month after sampling. Plasma lipids measurement was performed as reported previously [21]. Plasma concentrations 


\section{Kidney \\ Blood Pressure Research}

of lipoprotein(a) were determined by the Macra® Lp(a) Enzyme Linked Immunosorbent Assay (ELISA) kit (Trinity Biotech PLC, Bray, Ireland) [21]. Active renin was assayed without previous antihypertensive drug washout by chemiluminescence enzyme immunoassay (CLIA) and plasma aldosterone was assayed by radioimmunoassay in plasma samples that were obtained with patients in the sitting position [22]. Creatinine clearance was assessed by duplicate 24-hour collections and normalized for body surface area. Albuminuria was measured in duplicate 24-hour urine collections by immunoturbidimetric method using a commercial kit with an analyzer ALBT2 (Tina-quant Albumin Gen.2, Roche/Hitachi, Mannheim, Germany) [23]. The limit of detection was $3 \mathrm{mg} / \mathrm{l}$ and the coefficient of variation between $0.7 \%$ and $1.9 \%$.

\section{Statistical analysis}

Data are presented as mean \pm SD for variables with normal distribution and as median (inter-quartile range) for skewed variables. Normally distributed variables were compared with the Student's $t$ test. Variables with skewed distribution were analysed after logarithmic transformation. One-way analysis of variance was used to compare variables at different time-points after renal revascularization. Relationships between continuously distributed variables were examined by linear regression analysis. Two-tailed probability value of less than $5 \%$ was considered to indicate statistical significance. All data analyses were performed using Stata 9.2 (StataCorp LP, TX, USA).

\section{Results}

Five-hundred-sixty-eight patients with true resistant hypertension were screened at the clinic from January 2002 to December 2011. In 54 patients evidence of ARAS >70\% was detected by angiography. Among patients with ARAS, 2 had bilateral stenosis and the remaining 52 had unilateral stenosis of the main renal artery. The clinical characteristics and comorbidities of patients with ARAS are shown in Table 1. A relevant proportion of patients smoked and had dyslipidemia and/or diabetes. More than one third of patients had coronary artery and/or peripheral artery disease as comorbidities.

None of the patients was lost at follow-up. Both systolic $(-17 \mathrm{~mm} \mathrm{Hg}$; $11 \%$ ) and diastolic (-9 mm Hg; 9\%) blood pressure decreased significantly 3 weeks after renal artery stenting and further significant decrease was observed at six months (Table 2). At six months, blood pressure was normalized $\quad(<140 / 90$ $\mathrm{mm} \mathrm{Hg}$ ) in 36 (67\%) of 54 patients and the average number of antihypertensive drugs was significantly reduced from baseline (2.1 vs. 3.4, respectively). Blood pressure levels and the number of antihypertensive drugs remained comparable in

Table 1. Clinical characteristics of hypertensive patients with resistant hypertension and atherosclerotic renal artery stenosis. Values are expressed as mean $\pm \mathrm{SD}$

\begin{tabular}{lc}
\hline Characteristics & $\begin{array}{c}\text { Patients } \\
(\mathrm{n}=54)\end{array}$ \\
\hline Age, years [range] & $69 \pm 9[43-80]$ \\
Males, $\mathrm{n}(\%)$ & $35(65)$ \\
Body mass index, kg/m² & $28.0 \pm 5.5$ \\
Systolic blood pressure, mm Hg [range] & $161 \pm 20[125-200]$ \\
Diastolic blood pressure, mm Hg [range] & $97 \pm 13[70-130]$ \\
Pulse pressure, mm Hg & $57 \pm 15$ \\
Duration of hypertension, years [range] & $15 \pm 10[1-29]$ \\
Antihypertensive drugs, n [range] & $3.4 \pm 0.6[3-5]$ \\
Diuretics, n (\%) & $29(54)$ \\
Beta blockers, $\mathrm{n}(\%)$ & $32(59)$ \\
Calcium channel blockers, n (\%) & $29(54)$ \\
ACE inhibitors, $\mathrm{n}(\%)$ & $15(28)$ \\
Angiotensin II receptor blockers, n (\%) & $20(37)$ \\
Alpha blockers, n (\%) & $11(20)$ \\
Smokers, $\mathrm{n}(\%)$ & $17(31)$ \\
Diabetes, $\mathrm{n}(\%)$ & $12(22)$ \\
Dyslipidemia, $\mathrm{n}(\%)$ & $35(65)$ \\
Coronary artery disease, $\mathrm{n}(\%)$ & $18(33)$ \\
Cerebrovascular disease, n (\%) & $6(11)$ \\
Peripheral artery disease, $\mathrm{n}(\%)$ & $22(41)$ \\
\hline
\end{tabular}




\section{Kidney \\ Blood Pressure Research}

Table 2. Blood pressure, blood biochemistries, and renal function in 54 patients with resistant hypertension and atherosclerotic renal artery stenosis at baseline and during follow-up after renal artery stenting. Values are expressed as mean \pm SD. Comparisons were done by analysis of variance and $\mathrm{P}$ values indicate differences of variables across the study. UACR, urinary albumin/creatinine ratio; IQR, interquartile range

\begin{tabular}{lcc}
\hline & Baseline & 3 weeks \\
Variable & $161 \pm 20[125-200]$ & $144 \pm 15[125-165$ \\
Systolic blood pressure, mm Hg [range] & $97 \pm 13[70-130]$ & $88 \pm 10[60-105]$ \\
Diastolic blood pressure, mm Hg [range] & $3.4 \pm 0.6[0-5]$ & $2.0 \pm 1.6[0-4]$ \\
Antihypertensive drugs, n [range] & $104 \pm 33$ & - \\
Glucose, mg/dl & $180 \pm 40$ & - \\
Total cholesterol, mg/dl & $52 \pm 12$ & - \\
HDL-cholesterol, mg/dl & $103 \pm 32$ & - \\
LDL-cholesterol, mg/dl & $130 \pm 63$ & - \\
Trigycerides, mg/dl & $22 \pm 20$ & - \\
Lipoprotein(a), mg/dl & $6.1 \pm 1.3$ & - \\
Uric acid, mg/dl & $51 \pm 74$ & $15 \pm 14$ \\
Active renin, OUI/ml & $178 \pm 95$ & $153 \pm 93$ \\
Aldosterone, pg/ml & $1.25 \pm 0.38$ & $1.25 \pm 0.32$ \\
Creatinine, mg/dl & $81 \pm 48$ & $77 \pm 34$ \\
24-h creatinine clearance, ml/min/1.73 m ${ }^{2}$ & $119 \pm 39$ & $121 \pm 39$ \\
Urinary albumin excretion, mg/d & $84[53-148]$ & $91[59-162]$ \\
UACR, mg/g [IQR] & & \\
\hline
\end{tabular}

Table 3. Echocardiographic variables in 54 patients with resistant hypertension and atherosclerotic renal artery stenosis at baseline and during follow-up after renal artery stenting. Values are expressed as mean \pm SD. Comparisons were done by analysis of variance and P values indicate differences of variables across the study. LV, left ventricular

\begin{tabular}{lcccc}
\hline & Baseline & 6 months & 4 years & $\mathrm{P}$ \\
Variable & $51 \pm 7$ & $52 \pm 7$ & $51 \pm 5$ & 0.439 \\
LV end-diastolic diameter, mm & $32 \pm 6$ & $30 \pm 6$ & $31 \pm 5$ & 0.192 \\
LV end-systolic diameter, mm & $11.3 \pm 2.2$ & $10.9 \pm 1.9$ & $9.8 \pm 2.0$ & $<0.001$ \\
Interventricular septum, mm & $10.6 \pm 1.7$ & $10.6 \pm 1.8$ & $9.5 \pm 1.4$ & $<0.001$ \\
Posterior wall, mm & $205 \pm 76$ & $201 \pm 75$ & $186 \pm 50$ & 0.313 \\
LV mass, g & $111 \pm 37$ & $110 \pm 38$ & $101 \pm 26$ & 0.248 \\
Indexed LV mass, g/m ${ }^{2}$ & $0.429 \pm 0.081$ & $0.413 \pm 0.083$ & $0.378 \pm 0.077$ & 0.041 \\
Relative wall thickness, \% & $67 \pm 8$ & $66 \pm 8$ & $66 \pm 6$ & 0.720 \\
LV ejection fraction, \% & $38 \pm 7$ & $37 \pm 8$ & $40 \pm 8$ & 0.502 \\
LV fractional shortening, \% & $44 \pm 9$ & $45 \pm 7$ & $41 \pm 8$ & 0.029 \\
Left atrial diameter, mm & $0.83 \pm 0.28$ & $0.81 \pm 0.29$ & $0.96 \pm 0.34$ & 0.023 \\
E/A ratio & $234 \pm 68$ & $234 \pm 71$ & $237 \pm 70$ & 0.967 \\
E wave deceleration time, msec & $88 \pm 24$ & $90 \pm 25$ & $94 \pm 25$ & 0.439 \\
Isovolumic relaxation time, msec & & &
\end{tabular}

the long-term follow-up when blood pressure was normalized in $48(89 \%)$ patients. Plasma renin levels decreased significantly 3 weeks after renal revascularization and did not change significantly thereafter, whereas plasma aldosterone levels did not change significantly throughout the study. No significant changes were observed in plasma glucose, lipids, and uric acid levels during follow-up. After renal artery revascularization, 24-h creatinine clearance increased in 21 (39\%) patients, decreased in 28 (52\%) and remained stable in 


\section{Kidney Blood Pressure Research}

the remaining $5(9 \%)$ with an average value that had a small and nonsignificant decrease at the end of follow-up. Urinary albumin excretion did not change significantly in any patient throughout the study.

Echocardiographic variables of patients with ARAS that were measured at baseline and during follow-up are summarized in Table 3. No significant changes in LV internal dimensions, mass, geometry, systolic and diastolic function were observed at 6 months after renal artery revascularization. At the end of the long-term follow-up, interventricular septum and LV posterior wall thickness were significantly reduced and the average LV mass index was decreased but not significantly $\left(-10 \mathrm{~g} / \mathrm{m}^{2}\right)$. Also, the LV RWT was significantly decreased indicating reversal of the concentric pattern of LV geometry. No significant changes were observed in LV systolic function, whereas a significant reduction of left atrial diameter and increase in the E/A ratio indicated significant improvement of the LV diastolic function.

Univariate correlation analysis was used to examine the possible relationships between clinical (age, BMI, blood pressure, duration of hypertension) and biochemical (plasma glucose, lipids, uric acid, renin, aldosterone, creatinine clearance) variables that were measured at baseline and the changes in blood pressure, echocardiographic, and renal variables that were observed after renal artery revascularization. This analysis did not detect any significant relationships.

\section{Discussion}

Results of randomized clinical trials indicate that revascularization of ARAS does not confer any significant benefit over medical therapy [10,24]. However, these trials have been broadly criticized because of criteria used for patients' selection and, as suggested by many observational studies, timely identification and treatment of ARAS might modify the natural history of disease at least in some of these patients. The challenge is therefore to define which patients with ARAS will potentially benefit from renal artery revascularization in terms of renal and cardiovascular protection [25] and specifically designed observational investigations could provide insight in specific groups of patients. The present study has been the first designed specifically to investigate the effects of renal artery stenting in patients with resistant hypertension and hemodynamically significant ( $>70 \%)$ ARAS free of advanced renal failure and overt proteinuria. Patients were followed with a rigorous protocol with standardized antihypertensive medications and long-term follow-up providing evidence of significant and persistent benefit on blood pressure levels. Creatinine clearance and urinary albumin excretion remained unchanged after renal artery revascularization whereas, in the long-term, hypertension-related cardiac abnormalities were affected by treatment with significant decrease of LV wall thickness and concentric geometry, and improvement of LV diastolic function.

Previous retrospective and prospective observational studies reported the benefits of renal artery revascularization in terms of blood pressure decrease and renal protection in patients with ARAS $\geq 50 \%[26,27]$ even when this was associated with uncontrolled hypertension [28-30]. In a registry of 265 patients with angiographic evidence of ARAS $\geq 50 \%$, a subgroup of whom had uncontrolled systolic hypertension, average blood pressure fell in a follow-up of 2 years from 160/86 mm $\mathrm{Hg}$ to 135/75 mm $\mathrm{Hg}$ [28]. Approximately half of patients in the registry had improved glomerular filtration suggesting that renal revascularization could preserve renal function in a substantial proportion. The HERCULES trial examined prospectively 202 patients with ARAS, $70 \%$ of whom had uncontrolled blood pressure $(\geq 140 / 90 \mathrm{~mm} \mathrm{Hg}$ ) despite being on 3 or more antihypertensive agents [29]. In a 3-year follow-up, systolic blood pressure decreased by $16 \mathrm{~mm} \mathrm{Hg}$ with no changes in the average number of antihypertensive drugs. However, in a retrospective analysis of 32 patients with refractory hypertension and ARAS $\geq 50 \%$ who underwent renal artery revascularization, Ritchie et al. did not observe any benefit on mortality, cardiovascular 


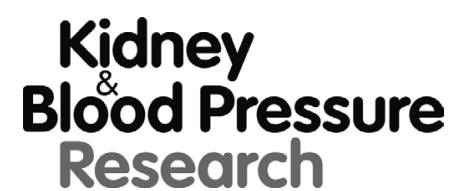

Kidney Blood Press Res 2017;42:774-783

\begin{tabular}{|l|l|}
\hline DOI: 10.1159/000484299 & (c) 2017 The Author(s). Published by S. Karger AG, Basel \\
\hline
\end{tabular}

events, or end-stage renal disease during a follow-up of 3.8 years, in comparison to patients treated conservatively [30]. Additional observational studies lasting from 6 to 15 months examined the renal outcome of renal artery revascularization of ARAS in patients without resistant hypertension and reported a significant decrease in blood pressure levels with no changes in glomerular filtration $[26,27]$ or urinary albumin excretion $[31,32]$. The results of the present prospective study further support the benefits on blood pressure control and preservation of renal function that can be obtained with renal artery stenting and extends the evidence to patients with hemodynamically significant ARAS who were identified because of resistant hypertension.

Cross-sectional investigations have demonstrated that hypertension-related cardiac abnormalities are significantly more frequent in patients with renal artery stenosis than matched patients with essential hypertension [33, 34]. Moreover, longitudinal assessment of cardiac structural and functional changes in patients with ARAS who were treated conservatively for 1 year demonstrated progression of these changes despite significant decrease in blood pressure [35]. Past studies addressed the effects of renal artery revascularization in hypertensive patients with renal artery stenosis (either ARAS or fibromuscular dysplasia) detected at angiography. Symonides et al. reported significant reduction of LV mass in 44 patients with either ARAS or fibromuscular dysplasia who underwent either surgical or percutaneous revascularization [31]. Similar reduction of LV mass was reported after renal artery stenting by Rzeznik et al [36]. and Zeller et al [37]. in a 1-year and 2-year follow-up study, respectively. Our study was conducted in patients with resistant hypertension and ARAS and demonstrates that successful renal artery stenting is followed, in the long-term, by reduction of LV mass that is associated with significant improvement of $\mathrm{LV}$ diastolic function.

Only a critical degree of renal artery stenosis can cause renal ischemia sufficient to activate the renin-angiotensin system and thereby hypertension, eventually leading to renal impairment. Although there is no established consensus on the degree of renal artery stenosis that justifies revascularization, the majority of previous observational and randomized studies used a 50\% diameter stenosis as inclusion criterion [10]. However, it is known that due to compensatory intrarenal hemodynamics occurrence of reduced renal perfusion pressure with subsequent activation of the renin-angiotensin system requires renal arterial narrowing of more than $70 \%$ [38]. For these reasons the present study included only patients with this degree of renal artery stenosis. Moreover, the evidence of a hemodynamically relevant stenosis in our patients was supported by the demonstration of highly significant reduction of plasma renin levels that despite use of antihypertensive agents, occurred 3 weeks after renal stenting.

Some limitations of this study need to be highlighted. First, this is an observational study and, as such, cannot provide any insight on the value of renal stenting in comparison to conservative treatment. Second, use of a clinic sample might limit the possibility to extend conclusions to a more general setting because of a possible bias due to the referral of patients to the source of care. Third, because we did not find any associations between clinical and biochemical variables measured at baseline and changes in blood pressure and echocardiographic variables observed after renal stenting, statistical limitations in detection of these associations due to the size of the study (beta-error) cannot be excluded. Last, systematic use of ambulatory blood pressure monitoring during follow-up would have been useful to obtain better assessment of blood pressure changes induced by treatment. The strengths of this study include inclusion of a selected population of patients with resistant hypertension and evidence of hemodynamically significant ARAS, the rigorous treatment protocol that was used after renal stenting, and the duration of follow-up. 


\section{Kidney Blood Pressure Research}

Catena et al.: Effects of Renal Revascularization

\section{Conclusion}

In patients with hypertension resistant to treatment and evidence of hemodynamically significant ARAS renal stenting decreases blood pressure, preserves renal function in a substantial proportion of patients, and improves LV structure and function in the longterm. These results indicate that negative results of randomized controlled trials of renal stenting performed in settings different from resistant hypertension should not withheld proper diagnostic evaluation for possible ARAS in these patients. Timely identification and correction of ARAS in patients with resistant hypertension could modify the natural history of the disease with significant benefits for both renal and cardiovascular outcomes.

\section{Disclosure Statement}

The authors have no Disclosure Statement related to the contents of this study to disclose.

\section{Acknowledgements}

This work was supported by a European Cooperation in the field Scientific and Technical Research (COST ADMIRE network, BM1301) grant to C.C. and by a research grant of the Pier Silverio Nassimbeni Foundation to C.C. and L.A.S.

\section{References}

1 Persell SD: Prevalence of resistant hypertension in the United States, 2003-2008 Hypertension 2011;57:1076-1080.

2 Daugherty SL, Powers JD, Magid DJ, Tavel HM, Masoudi FA, Maragolis KL, O’Connor PJ, Selby JV, Ho PM: Incidence and prognosis of resistant hypertension in hypertensive patients. Circulation 2012;125:16351642.

3 Catena C, Novello M, Lapenna R, Baroselli S, Colussi GL, Nadalini E, Favret G, Cavarape A, Soardo G, Sechi LA: New risk factors for atherosclerosis in hypertension: focus on the prothrombotic state and lipoprotein(a). J Hypertens 2005;23:1617-1631.

-4 Fagard RH: Resistant hypertension. Heart 2012;98:254-261.

$>5$ Hansen KJ, Edwards MS, Craven TE, Cherr GS, Jackson SA, Appel RG, Burke GL, Dean RH: Prevalence of renovascular disease in the elderly: a population-based study. J Vasc Surg 2002;36:443-451.

-6 Cohen MG, Pascua JA, Garcia-Ben M, Rojas-Matas CA, Gabay JM, Berrocal DH, Tan WA, Stouffer GA, Montoya M, Fernandez AD, Halac ME, Grinfeld LR: A simple prediction rule for significant renal artery stenosis in patients undergoing cardiac catheterization. Am Heart J 2005;150:1204-1211.

7 Safian RD, Textor SC: Renal-artery stenosis. N Engl J Med 2001;344:431-442

-8 Van Ampting JM, Penne EL, Beek FJ, Koomans HA, Boer WH, Beutler JJ: Prevalence of atherosclerosis renal artery stenosis in patients starting dialysis. Nephrol Dial Transplant 2003;18:1147-1151.

-9 Vassallo D, Green D, Ritchie J, Chrysochou C, Blunt J, Kalra PA: Three decades of atherosclerotic renovascular disease management - changing outcomes in an observational study. Kidney Blood Press Res 2016;41:325-334.

10 Van der Niepen P, Rossignol P, Lengele J-P, Berra E, Sarafidis P, Persu A: Renal artery stenosis in patients with resistant hypertension: stent it or not? Curr Hypertens Rep 2017;19:5.

11 Bavishi C, de Leeuw PW, Messerli FH: Atherosclerotic renal artery stenosis and hypertension: pragmatism, pitfalls, and perspectives. Am J Med 2016;129:635.e5-635.e14.

12 Benjamin MM, Fazel P, Filardo G, Choi JW, Stoler RC: Prevalence and risk factors of renal artery stenosis in patients with resistant hypertension. Am J Cardiol 2014;113:687-690. 


\section{Kidney \\ Blood Pressure Research}

13 Mancia G, De Backer G, Dominczak A, Cifkova R, Fagard R, Germanò G, Grassi G, Heagerty AM, Kjeldsen SE, Laurent S, Narkiewicz K, Ruilope L, Rynkiewicz A, Schmieder RE, Boudier HA, Zanchetti A, Vahanian A, Camm J, De Caterina R, Dean V, Dickstein K, Filippatos G, Funck-Brentano C, Hellemans I, Kristensen SD, McGregor K, Sechtem U, Silber S, Tendera M, Widimsky P, Zamorano JL, Erdine S, Kiowski W, Lindholm LH, Viigimaa M, Adamopoulos S, Agabiti-Rosei E, Ambrosioni E, Bertomeu V, Clement D, Erdine S, Farsang C, Gaita D, Lip G, Mallion JM, Manolis AJ, Nilsson PM, O’Brien E, Ponikowski P, Redon J, Ruschitzka F, Tamargo J, van Zwieten P, Waeber B, Williams B: 2007 ESH-ESC practice guidelines for the management of arterial hypertension. J Hypertens 2007;25:1105-1187.

14 Sechi LA, Catena C, Casaccio D, Zingaro L: Lipoprotein(a), haemostatic variables and cardiovascular damage in hypertensive patients. J Hypertens 2000;18:709-716.

15 Catena C, Colussi GL, Novello M, Fagotto V, Sechi LA: Intrarenal vascular resistance is associated with a prothrombotic state in hypertensive patients. Kidney Blood Press Res 2017;41:929-936.

16 Colussi GL, Catena C, Baroselli S, Nadalini E, Lapenna R, Chiuch A, Sechi LA: Omega-3 fatty acids: from biochemistry to their clinical use in the prevention of cardiovascular disease. Recent Patents Cardiovasc Drug Discov 2007;2:13-21.

17 Sechi LA, Novello M, Colussi GL, Di Fabio A, Chiuch A, Nadalini E, Casanova-Borca A, Uzzau A, Catena C: Relationship of plasma renin with a prothrombotic state in hypertension: relevance for organ damage. Am J Hypertens 2008; 21:1347-1353.

18 Catena C, Colussi GL, Nait F, Capobianco F, Sechi LA: Plasma lipoprotein(a) levels and atherosclerotic renal artery stenosis in hypertensive patients. Kidney Blood Press Res 2015;40:166-175.

19 Sechi LA, Zingaro L, Catena C, De Marchi S: Increased fibrinogen levels and hemostatic abnormalities in patients with arteriolar nephrosclerosis: association with cardiovascular events. Thromb Haemost 2000;84:565-570.

20 Catena C, Colussi GL, Marzano L, Sechi LA: Predictive factors of left ventricular mass changes after treatment of primary aldosteronism. Horm Metab Res 2012;44:188-193.

-21 Catena C, Novello M, Dotto L, De Marchi S, Sechi LA: Serum lipoprotein(a) concentrations and alcohol consumption in hypertension: possible relevance for cardiovascular damage. J Hypertens 2003;21:281288.

22 Catena C, Colussi, GL, Valeri M, Sechi LA: Association of aldosterone with left ventricular mass in hypertension: interaction with plasma fibrinogen levels. Am J Hypertens 2013;26:111-117.

23 Sechi LA, Zingaro L, Catena C, Perin A, De Marchi S, Bartoli E: Lipoprotein(a) and apolipoprotein(a) isoforms and proteinuria in patients with moderate renal failure. Kidney Int 1999;56:1049-1057.

24 Cooper CJ, Murphy TP, Cutlip DE, Jamerson K, Henrich W, Reid DM, Cohen DJ, Matsumoto AH, Steffes M, Jaff MR, Prince MR, Lewis EF, Tuttle KR, Shapiro JI, Rundback JH, Massaro JM, D’Agostino RB, Dworkin LD for the CORAL Investigators: Stenting and medical therapy for atherosclerotic renal-artery stenosis. New Engl J Med 2014;370:13-22.

25 Textor SC, Misra S: Does clinical artery stenting prevent clinical events? CLin J Am Soc Nephrol 2016;11:1125-1127.

-26 Burket MW, Cooper CJ, Kennedy DJ, Brewster PS, Ansel GM, Moore JA, Venkatesan J, Henrich WL: Renal angioplasty and stent placement: predictors of a favorable outcome. Am Heart J 2000;139:64-71.

-27 Prajapati JS, Jain SR, Joshi H, Shah S,Sharma K, Sahoo S, Virparia K, Thakkar A: Response of blood pressure after percutaneous transluminal renal artery angioplasty and stenting. World J Cardiol 2013;5:247-253.

-28 Milewski K, Fil W, Buszman P, Janik M, Wanha W, Martin T, Krol M, Gorycki B, Wiernek S, Krzych L, Kiesz RS, Wojakowski W, Buszman P: Renal artery stenting associated with improvement of renal function and blood pressure control in long-term follow-up. Kidney Bloo Press Res 2016;41:278-287.

-29 Chrysant GS, Bates MC, Sullivan TM, Bachinsky WB, Popma JJ, OPeng L, Omran HL, Jaff MR, HERCULES investigators: Proper patient selection yields significant and sustained reduction in systolic blood pressure following renal artery stenting in patients with uncontrolled hypertension: long-term results from the HERCULES trial. J Clin Hypertens 2014;16:497-503.

-30 Ritchie J, Green D, Chrysochou C, Chalmers N, Foley RN, Kaira PA: High-risk clinical presentations in atherosclerotic renovascular disease: prognosis and response to renal artery revascularization. Am J Kidey Dis 2014;63:186-197. 


\section{Kidney

-31 Symonides B, Chodakowska J, Januszewicz A, Lapinski M, Januszewicz M, Rowinski O, Szmidt J, Kuch-Wocial A, Kurzyna M, Malek G, Berent H, Szmigielski C, Januszewicz W: Effects of the correction of renal artery stenosis on blood pressure, renal function and left ventricular morphology. Blood Press 1999;8:141-150.

-32 Kadziela J, Prejbisz A, Michalowska I, Adamczak M, Warchol-Celinska E, Pregowska-Chwala B, JanuszewiczM, Wiecek A, Januszewicz A, Witkowski A: Relationship between hemodynamic parameters of renal artery stenosis and the changes of kidney function after renal artery stenting in patients with hypertension and preserved renal function. Blood Press 2015;24:30-34.

-33 Wu S, Polavarapu N, Stouffer GA: Left ventricular hypertrophy in patients with renal artery stenosis. Am J Med Sci 2006;332:334-338.

34 Hoshida S, Shinoda Y, Inui H, Hosoi R, Teranishi F, Asaoka N, Sugitani T, Hamasaki T: Difference in left ventricular mass index between hypertensive patients with and without renal artery stenosis by propensity score analysis. J Clin Hypertens 2014;16:606-611.

35 Wright JR, Shurrab AE, Cooper A, Kalra PR, Foley RN, Kalra PA: Progression of cardiac dysfunction in patients with atherosclerotic renovascular disease. QJM 2009;102:695-704.

-36 Rzeznik D, Przewlocki T, Kablak-Ziembicka A, Kozaneki A, Roslawiecka A, Lach J, Tracz W, Podolec P: Effect of renal artery revascularization on left ventricular hypertrophy, diastolic function, blood pressure, and the one-year outcome. J Vasc Surg 2011;53:692-697.

-37 Zeller T, Restan A, Schwarzwalder U, Muller C, Frank U, Burgelin K, Sixt S, Schwarz T, Noory E, Neumann FJ: Regression of left ventricular hypertrophy following stenting of renal artery stenosis. J Endovasc Ther 2007;14:189-197.

-38 De Bruyne B, Manoharan G, Pijls NH, Verhamme K, Madaric J, Bartunek J, Vanderheyden M, Heyndricks GR: Assessment of renal artery stenosis severity by pressure gradient measurements. J Am Coll Cardiol 2006;48:1851-1855. 